\title{
Numerical approximation to a solution of the modified regularized long wave equation using quintic B-splines
}

\author{
Seydi Battal Gazi Karakoc ${ }^{1}$, Nuri Murat Yagmurlu ${ }^{2 *}$ and Yusuf Ucar ${ }^{2}$
}

\section{${ }^{*}$ Correspondence:}

murat.yagmurlu@inonu.edu.tr

${ }^{2}$ Department of Mathematics,

Faculty of Science and Art, Inönü

University, Malatya, 44280, Turkey

Full list of author information is

available at the end of the article

\begin{abstract}
In this work, a numerical solution of the modified regularized long wave (MRLW) equation is obtained by the method based on collocation of quintic B-splines over the finite elements. A linear stability analysis shows that the numerical scheme based on Von Neumann approximation theory is unconditionally stable. Test problems including the solitary wave motion, the interaction of two and three solitary waves and the Maxwellian initial condition are solved to validate the proposed method by calculating error norms $L_{2}$ and $L_{\infty}$ that are found to be marginally accurate and efficient. The three invariants of the motion have been calculated to determine the conservation properties of the scheme. The obtained results are compared with other earlier results.
\end{abstract}

MSC: $97 \mathrm{~N} 40 ; 65 \mathrm{~N} 30 ; 65 \mathrm{D} 07 ; 76 \mathrm{~B} 25 ; 74 \mathrm{~S} 05$

Keywords: MRLW equation; collocation; finite element method; B-spline; solitary waves

\section{Introduction}

The modified regularized long wave (MRLW) equation, based upon the regularized long wave (RLW) equation,

$$
U_{t}+U_{x}+\delta U U_{x}-\mu U_{x x t}=0
$$

which was proposed at first by Peregrine [1] to describe the development of an undular bore, has the form

$$
U_{t}+U_{x}+6 U^{2} U_{x}-\mu U_{x x t}=0
$$

where $\delta$ and $\mu$ are positive parameters and the subscripts $x$ and $t$ denote the differentiation. The RLW equation is one of the best known partial differential equations because it describes a large number of important physical phenomena with weak nonlinearity and dispersion waves such as magneto hydrodynamic and ion-acoustic waves in plasma, phonon packets in non-linear crystals, the transverse waves in shallow water, rotating flow down a tube and pressure waves in liquid-gas bubble mixtures. Bona and Pryant [2] have studied the existence and uniqueness of the equation. Benjamin et al. [3] have proposed the RLW equation as a numerically superior modification of the Korteweg de-Vries (KdV) equation.

\section{楚 Springer}

(c) 2013 Karakoc et al.: licensee Springer. This is an Open Access article distributed under the terms of the Creative Commons Attribution License (http://creativecommons.org/licenses/by/2.0), which permits unrestricted use, distribution, and reproduction in any medium, provided the original work is properly cited. 
This superiority arises because, unlike the KdV equation, the dispersion relation associated with the linearized RLW equation yields the frequency that is bounded for large wave numbers [4]. But they have found an analytical solution of the RLW equation under the restricted initial and boundary conditions. So, various numerical techniques have been introduced to solve the equation. These include the finite difference [5-7], finite element [8-22], Fourier pseudo-spectral [23] methods and the meshfree method [24]. One of the special properties of the equation is that the solutions may exhibit solitons whose magnitudes, shapes and velocities are not changed after the collision. The RLW equation is a special case of the generalized long wave (GRLW) equation having the form

$$
U_{t}+U_{x}+\delta U^{p} U_{x}-\mu U_{x x t}=0
$$

where $p$ is a positive integer. Zhang [25] has used the finite difference method to solve the GRLW equation for a Cauchy problem. The quasilinearization method based on finite differences was used by Ramos [26] for solving the GRLW equation. Kaya et al. [27] have also studied the GRLW equation with the Adomian decomposition method. Roshan [28] has solved the GRLW equation numerically by the Petrov-Galerkin method using a linear hat function as the trial function and a quintic B-spline function as the test function. Gardner et al. [29] have developed a collocation solution to the MRLW equation using quintic Bsplines finite elements. Khalifa et al. [30,31] have obtained the numerical solutions of the MRLW equation using the finite difference method and the cubic B-spline collocation finite element method. Solutions based on the collocation method with quadratic B-spline finite elements and the central finite difference method for time have been investigated by Raslan [32]. Raslan and Hassan [33] have solved the MRLW equation by the collocation finite element method using quadratic, cubic, quartic and quintic B-splines to obtain the numerical solutions of a single solitary wave. Fazal-i-Haq et al. [34] have designed a numerical scheme based on the quartic B-spline collocation method for the numerical solution of the MRLW equation. Ali [35] has formulated a classical radial basis functions (RBFs) collocation method for solving the MRLW equation. In this paper, we have obtained a type of the quintic B-spline collocation procedure in which a nonlinear term in the equation is linearized by using the form introduced by Rubin and Graves [36] to solve the MRLW equation. The proposed method is shown to represent accurately the migration of a single solitary wave. Then the interaction of two and three solitary waves and the Maxwellian initial condition are studied. The linear stability analysis based on the Von Neumann method is also investigated.

\section{Quintic B-spline finite element solution}

Let us consider MRLW equation (2) with the following initial,

$$
U(x, 0)=f(x), \quad a \leq x \leq b,
$$

and boundary conditions:

$$
\begin{aligned}
& U(a, t)=0, \quad U(b, t)=0, \\
& U_{x}(a, t)=0, \quad U_{x}(b, t)=0, \\
& U_{x x}(a, t)=0, \quad U_{x x}(b, t)=0, \quad t>0 .
\end{aligned}
$$


For the numerical calculation, the solution domain of the problem is restricted over an interval $a \leq x \leq b$. The interval is partitioned into uniformly-sized finite elements of length $h$ by the knots $x_{m}$ such that $a=x_{0}<x_{1}<\cdots<x_{N}=b$. The set of quintic B-spline functions $\left\{\phi_{-2}(x), \phi_{-1}(x), \ldots, \phi_{N+1}(x), \phi_{N+2}(x)\right\}$ forms a basis over the problem domain $[a, b]$. We seek the numerical solution $U_{N}(x, t)$ to the exact solution $U(x, t)$ in the form of

$$
U_{N}(x, t)=\sum_{j=-2}^{N+2} \phi_{j}(x) \delta_{j}(t)
$$

where $\delta_{j}(t)$ are time dependent parameters to be determined from the boundary and collocation conditions.

Quintic B-splines $\phi_{m}(x)(m=-2(1) N+2)$, at the knots $x_{m}$ are defined over the interval $[a, b]$ by [37].

$$
\phi_{m}(x)=\frac{1}{h^{5}} \begin{cases}\left(x-x_{m-3}\right)^{5}, & {\left[x_{m-3}, x_{m-2}\right],} \\ \left(x-x_{m-3}\right)^{5}-6\left(x-x_{m-2}\right)^{5}, & {\left[x_{m-2}, x_{m-1}\right],} \\ \left(x-x_{m-3}\right)^{5}-6\left(x-x_{m-2}\right)^{5}+15\left(x-x_{m-1}\right)^{5}, & {\left[x_{m-1}, x_{m}\right],} \\ \left(x-x_{m-3}\right)^{5}-6\left(x-x_{m-2}\right)^{5}+15\left(x-x_{m-1}\right)^{5} & \\ -20\left(x-x_{m}\right)^{5}, & {\left[x_{m}, x_{m+1}\right]} \\ \left(x-x_{m-3}\right)^{5}-6\left(x-x_{m-2}\right)^{5}+15\left(x-x_{m-1}\right)^{5} & \\ -20\left(x-x_{m}\right)^{5}+15\left(x-x_{m+1}\right)^{5}, & {\left[x_{m+1}, x_{m+2}\right]} \\ \left(x-x_{m-3}\right)^{5}-6\left(x-x_{m-2}\right)^{5}+15\left(x-x_{m-1}\right)^{5} & \\ -20\left(x-x_{m}\right)^{5}+15\left(x-x_{m+1}\right)^{5}-6\left(x-x_{m+2}\right)^{5}, & {\left[x_{m+2}, x_{m+3}\right]} \\ 0, & \text { otherwise. }\end{cases}
$$

Each quintic B-spline covers six elements so that each element $\left[x_{m}, x_{m+1}\right]$ is covered by six B-splines. Substituting trial function (7) into Eq. (6), the nodal values of $U, U^{\prime}, U^{\prime \prime}$ at the knots $x_{m}$ are obtained in terms of the element parameters $\delta_{m}$ by

$$
\begin{aligned}
& U_{N}\left(x_{m}, t\right)=U_{m}=\delta_{m-2}+26 \delta_{m-1}+66 \delta_{m}+26 \delta_{m+1}+\delta_{m+2} \\
& U_{m}^{\prime}=\frac{5}{h}\left(-\delta_{m-2}-10 \delta_{m-1}+10 \delta_{m+1}+\delta_{m+2}\right) \\
& U_{m}^{\prime \prime}=\frac{20}{h^{2}}\left(\delta_{m-2}+2 \delta_{m-1}-6 \delta_{m}+2 \delta_{m+1}+\delta_{m+2}\right)
\end{aligned}
$$

where the symbols ' and " represent first and second differentiation with respect to $x$, respectively. The splines $\phi_{m}(x)$ and their four principle derivatives vanish outside the interval $\left[x_{m-3}, x_{m+3}\right]$.

Using a first-order forward difference formula for the time derivative of the $U$ and Crank-Nicolson approximation for the space derivatives $U_{x}$ and $U_{x x}$ in Eq. (2) leads to

$$
\frac{U^{n+1}-U^{n}}{\Delta t}+\frac{U_{x}^{n+1}+U_{x}^{n}}{2}+6 \frac{\left(U^{2} U_{x}\right)^{n+1}+\left(U^{2} U_{x}\right)^{n}}{2}-\mu \frac{U_{x x}^{n+1}-U_{x x}^{n}}{\Delta t}=0
$$

Now, if we apply a linearization technique similar to the one first introduced by Rubin and Graves [36] to Eq. (9),

$$
\left(U^{2} U_{x}\right)^{n+1}=U^{n+1} U^{n} U_{x}^{n}+U^{n} U^{n+1} U_{x}^{n}+U^{n} U^{n} U_{x}^{n+1}-2 U^{n} U^{n} U_{x}^{n},
$$


we obtain

$$
\begin{gathered}
U^{n+1}+\frac{\Delta t}{2} U_{x}^{n+1}+3 \Delta t\left(U^{n+1} U^{n} U_{x}^{n}+U^{n} U^{n+1} U_{x}^{n}+U^{n} U^{n} U_{x}^{n+1}\right)-\mu U_{x x}^{n+1} \\
=U^{n}-\frac{\Delta t}{2} U_{x}^{n}-3 \Delta t\left(U^{2} U_{x}\right)^{n}-\mu U_{x x}^{n}+6 \Delta t\left(U^{n} U^{n} U_{x}^{n}\right) .
\end{gathered}
$$

If we substitute the nodal values of $U, U_{x}$ and $U_{x x}$ given by (8) into (10), we obtain the following iterative system:

$$
\begin{aligned}
\delta_{m-2}^{n+1}( & \left.-\alpha_{1}+2 \alpha_{2}-\alpha_{3}-\alpha_{4}\right)+\delta_{m-1}^{n+1}\left(26-10 \alpha_{1}+52 \alpha_{2}-10 \alpha_{3}-2 \alpha_{4}\right) \\
& +\delta_{m}^{n+1}\left(66+132 \alpha_{2}+6 \alpha_{4}\right)+\delta_{m+1}^{n+1}\left(26+10 \alpha_{1}+52 \alpha_{2}+10 \alpha_{3}-2 \alpha_{4}\right) \\
& +\delta_{m+2}^{n+1}\left(1+\alpha_{1}+2 \alpha_{2}+\alpha_{3}-\alpha_{4}\right) \\
= & \delta_{m-2}^{n}\left(1+\alpha_{1}+\alpha_{2}-\alpha_{4}\right)+\delta_{m-1}^{n}\left(26+10 \alpha_{1}+26 \alpha_{2}-2 \alpha_{4}\right)+\delta_{m}^{n}\left(66+66 \alpha_{2}+6 \alpha_{4}\right) \\
& +\delta_{m+1}^{n}\left(26-10 \alpha_{1}+26 \alpha_{2}-2 \alpha_{4}\right)+\delta_{m+2}^{n}\left(1-\alpha_{1}+\alpha_{2}-\alpha_{4}\right), \quad m=0(1) N,
\end{aligned}
$$

where

$$
\begin{aligned}
& \alpha_{1}=\frac{5 \Delta t}{2 h}, \\
& \alpha_{2}=\frac{15 \Delta t}{h}\left(\delta_{m-2}^{n}+26 \delta_{m-1}^{n}+66 \delta_{m}^{n}+26 \delta_{m+1}^{n}+\delta_{m+2}^{n}\right)\left(-\delta_{m-2}^{n}-10 \delta_{m-1}^{n}+10 \delta_{m+1}^{n}+\delta_{m+2}^{n}\right), \\
& \alpha_{3}=\frac{15 \Delta t}{h}\left(\delta_{m-2}^{n}+26 \delta_{m-1}^{n}+66 \delta_{m}^{n}+26 \delta_{m+1}^{n}+\delta_{m+2}^{n}\right)^{2}, \\
& \alpha_{4}=\frac{20 \mu}{h^{2}} .
\end{aligned}
$$

This newly obtained iterative system (10) consists of $N+1$ linear equations including $N+5$ unknown parameters $\left(\delta_{-2}, \delta_{-1}, \ldots, \delta_{N+1}, \delta_{N+2}\right)^{T}$. To obtain a unique solution to this system, we need four additional constraints. These are obtained from the boundary conditions $U(a, t)=U(b, t)=0$ and $U_{x}(a, t)=U_{x}(b, t)=0$ and can be used to eliminate $\delta_{-2}, \delta_{-1}$ and $\delta_{N+1}, \delta_{N+2}$ from system (10), which then becomes a matrix equation for the $N+1$ unknowns $\mathbf{d}=\left(\delta_{0}, \delta_{1}, \ldots, \delta_{N}\right)^{T}$ of the form

$$
\operatorname{Ad}^{\mathbf{n}+1}=B \mathbf{d}^{\mathbf{n}}
$$

The matrices $\mathbf{A}$ and $\mathbf{B}$ are pentagonal $(N+1) \times(N+1)$ matrices given as

$$
\mathbf{A}=\left[\begin{array}{cccccccc}
a_{11} & a_{12} & a_{13} & & & & & \\
a_{21} & a_{22} & a_{23} & a_{24} & & & \\
& & \ddots & & & & \\
& a_{m, m-2} & a_{m, m-1} & a_{m, m} & a_{m, m+1} & a_{m, m+2} & & \\
& & & & \ddots & & & \\
& & & & a_{n, n-2} & a_{n, n-1} & a_{n, n} & a_{n, n+1} \\
& & & & & a_{n+1, n-1} & a_{n+1, n} & a_{n+1, n+1}
\end{array}\right],
$$

$$
m=3(1) n-1
$$




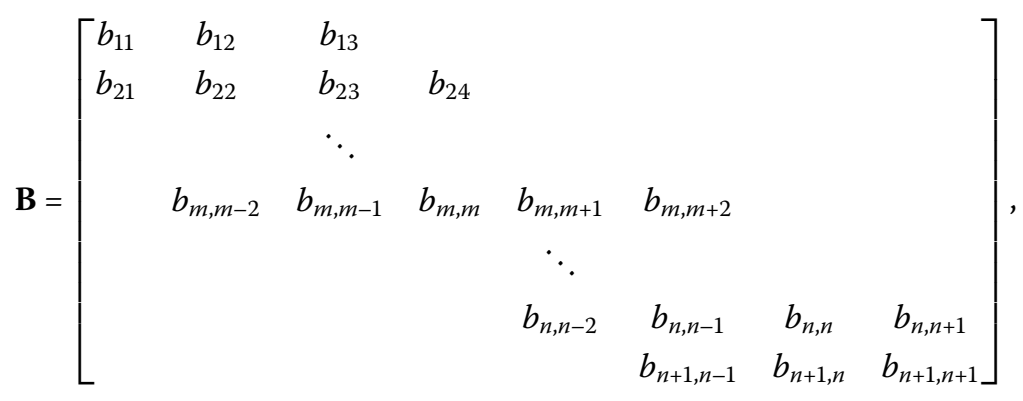

$m=3(1) n-1$

where

$$
\begin{array}{ll}
a_{11}=-27 \alpha_{4}, & b_{11}=-27 \alpha_{4}, \\
a_{12}=-30 \alpha_{4}, & b_{12}=-30 \alpha_{4}, \\
a_{13}=-3 \alpha_{4}, & b_{13}=-3 \alpha_{4}, \\
a_{21}=\frac{175}{8}-\frac{47}{8} \alpha_{1}+\frac{175}{4} \alpha_{2}-\frac{47}{8} \alpha_{3}+\frac{17}{8} \alpha_{4}, & b_{21}=\frac{175}{8}+\frac{47}{8} \alpha_{1}+\frac{175}{8} \alpha_{2}+\frac{17}{8} \alpha_{4}, \\
a_{22}=\frac{255}{4}+\frac{9}{4} \alpha_{1}+\frac{255}{2} \alpha_{2}+\frac{9}{4} \alpha_{3}+\frac{33}{4} \alpha_{4}, & b_{22}=\frac{255}{4}-\frac{9}{4} \alpha_{1}+\frac{255}{4} \alpha_{2}+\frac{33}{4} \alpha_{4}, \\
a_{23}=\frac{207}{8}+\frac{81}{8} \alpha_{1}+\frac{207}{4} \alpha_{2}+\frac{81}{8} \alpha_{3}-\frac{15}{8} \alpha_{4}, & b_{23}=\frac{207}{8}-\frac{81}{8} \alpha_{1}+\frac{207}{8} \alpha_{2}-\frac{15}{8} \alpha_{4}, \\
a_{24}=1+\alpha_{1}+2 \alpha_{2}+\alpha_{3}-\alpha_{4}, & b_{24}=1-\alpha_{1}+\alpha_{2}-\alpha_{4}, \\
a_{m, m-2}=1-\alpha_{1}+2 \alpha_{2}-\alpha_{3}-\alpha_{4}, & b_{m, m-2}=1+\alpha_{1}+\alpha_{2}-\alpha_{4}, \\
a_{m, m-1}=26-10 \alpha_{1}+52 \alpha_{2}-10 \alpha_{3}-2 \alpha_{4}, & b_{m, m-1}=26+10 \alpha_{1}+26 \alpha_{2}-2 \alpha_{4}, \\
a_{m, m}=66+132 \alpha_{2}+6 \alpha_{4}, & b_{m, m}=66+66 \alpha_{2}+6 \alpha_{4}, \\
a_{m, m+1}=26+10 \alpha_{1}+52 \alpha_{2}+10 \alpha_{3}-2 \alpha_{4}, & b_{m, m+1}=26-10 \alpha_{1}+26 \alpha_{2}-2 \alpha_{4}, \\
a_{m, m+2}=1+\alpha_{1}+2 \alpha_{2}+\alpha_{3}-\alpha_{4}, & b_{m, m+2}=1-\alpha_{1}+\alpha_{2}-\alpha_{4}, \\
m=3(1) n-1, & \\
a_{n-1, n-3}=1-\alpha_{1}+2 \alpha_{2}-\alpha_{3}-\alpha_{4}, & \\
a_{n-1, n-2}=\frac{207}{8}-\frac{81}{8} \alpha_{1}+\frac{207}{4} \alpha_{2}-\frac{81}{8} \alpha_{3}-\frac{15}{8} \alpha_{4}, & b_{n-1, n-2}=\frac{207}{8}+\frac{81}{8} \alpha_{1}+\frac{207}{8} \alpha_{2}-\frac{15}{8} \alpha_{4}, \\
a_{n-1, n-1}=\frac{255}{4}-\frac{9}{4} \alpha_{1}+\frac{255}{2} \alpha_{2}-\frac{9}{4} \alpha_{3}+\frac{33}{4} \alpha_{4}, & b_{n-1, n-1}=\frac{255}{4}+\frac{9}{4} \alpha_{1}+\frac{255}{4} \alpha_{2}+\frac{33}{4} \alpha_{4}, \\
a_{n-1, n}=\frac{175}{8}+\frac{47}{8} \alpha_{1}+\frac{175}{4} \alpha_{2}+\frac{47}{8} \alpha_{3}+\frac{17}{8} \alpha_{4}, & b_{n-1, n}=\frac{175}{8}-\frac{47}{8} \alpha_{1}+\frac{175}{8} \alpha_{2}+\frac{17}{8} \alpha_{4}, \\
a_{n, n-2}=-3 \alpha_{4}, & b_{n, n-2}=-3 \alpha_{4}, \\
a_{n, n-1}=-30 \alpha_{4}, & b_{n, n-1}=-30 \alpha_{4}, \\
a_{n, n}=-27 \alpha_{4}, & b_{n, n}=-27 \alpha_{4} . \\
& \\
m & \\
&
\end{array}
$$

To proceed with iterative formula (11), we need the initial vector $d^{0}$ which is determined from the initial and boundary conditions. For this purpose, approximation (6) must be rewritten for the initial condition as

$$
U_{N}(x, 0)=\sum_{m=-2}^{N+2} \delta_{m}(0) \phi_{m}(x)
$$

where the $\delta_{m}$ 's are unknown element parameters. Now, if we require the initial numerical approximation $U_{N}(x, 0)$ to satisfy the following boundary conditions to eliminate $\delta_{-1}$ 
and $\delta_{N+1}$ :

$$
\begin{aligned}
& U_{N}(x, 0)=U\left(x_{m}, 0\right), \quad m=0,1, \ldots, N, \\
& \left(U_{N}\right)_{x}(a, 0)=0, \quad\left(U_{N}\right)_{x}(b, 0)=0, \\
& \left(U_{N}\right)_{x x}(a, 0)=0, \quad\left(U_{N}\right)_{x x}(b, 0)=0,
\end{aligned}
$$

we obtain the following matrix form for the initial vector $\mathbf{d}^{0}$ :

$$
\mathbf{W} \mathbf{d}^{0}=\mathbf{b},
$$

where

$$
\begin{aligned}
& \mathbf{W}=\left[\begin{array}{cccccccc}
54 & 60 & 6 & & & & & \\
25.25 & 67.50 & 26.25 & 1 & & & & \\
1 & 26 & 66 & 26 & 1 & & & \\
& 1 & 26 & 66 & 26 & 1 & & \\
& & & & \ddots & & & \\
& & & 1 & 26 & 66 & 26 & 1 \\
& & & & 1 & 26.25 & 67.50 & 25.25 \\
& & & & 6 & 60 & 54
\end{array}\right], \\
& \mathbf{d}^{0}=\left(\delta_{0}, \delta_{1}, \delta_{2}, \ldots, \delta_{N-2}, \delta_{N-1}, \delta_{N}\right)^{T} \\
&
\end{aligned}
$$

and

$$
\mathbf{b}=\left(U\left(x_{0}, 0\right), U\left(x_{1}, 0\right), U\left(x_{2}, 0\right), \ldots, U\left(x_{N-2}, 0\right), U\left(x_{N-1}, 0\right), U\left(x_{N}, 0\right)\right)^{T} .
$$

\subsection{A linear stability analysis}

The stability analysis is based on the Von Neumann theory in which the growth factor of a typical Fourier mode is defined as

$$
\delta_{j}^{n}=\widehat{\zeta}^{n} e^{i j k h}
$$

where $k$ is a mode number and $h$ is the element size. The non-linear term $U^{2} U_{x}$ of the MRLW equation cannot be handled by the Fourier mode method. Thus, this term is linearized by making the quantity $U^{2}$ in the nonlinear term a local constant such as $Z_{m}$. Then substituting Eq. (15) into system (10) gives

$$
\widehat{\zeta}^{n+1}=g \widehat{\zeta}^{n}
$$

where $g$ is the growth factor.

Now, we identify the collocation points with the knots and use Eq. (8) to evaluate $U_{m}$ and its necessary space derivatives and substitute into Eq. (2) to obtain the following equa- 
tion:

$$
\begin{aligned}
\dot{\delta}_{m-2} & +26 \dot{\delta}_{m-1}+66 \dot{\delta}_{m}+26 \dot{\delta}_{m+1}+26 \dot{\delta}_{m+2} \\
& +\frac{5}{h}\left(1+6 Z_{m}\right)\left(-\delta_{m-2}-10 \delta_{m-1}+10 \delta_{m+1}+\delta_{m+2}\right) \\
& -\frac{20 \mu}{h^{2}}\left(\dot{\delta}_{m-2}+2 \dot{\delta}_{m-1}-6 \dot{\delta}_{m}+2 \dot{\delta}_{m+1}+26 \dot{\delta}_{m+2}\right)=0 .
\end{aligned}
$$

Here - denotes derivative with respect to time. If time parameters $\delta_{i}$ 's and their time derivatives $\dot{\delta}_{i}$ 's in Eq. (17) are discretized by the Crank-Nicolson formula and usual forward finite difference approximation, respectively:

$$
\delta_{i}=\frac{\delta^{n}+\delta^{n+1}}{2}, \quad \dot{\delta}_{i}=\frac{\delta^{n+1}-\delta^{n}}{\Delta t}
$$

we obtain a recurrence relationship between two time levels $n$ and $n+1$ relating two unknown parameters $\delta_{i}^{n+1}, \delta_{i}^{n}$ for $i=m-2, m-1, \ldots, m+1, m+2$

$$
\begin{aligned}
& \gamma_{1} \delta_{m-2}^{n+1}+\gamma_{2} \delta_{m-1}^{n+1}+\gamma_{3} \delta_{m}^{n+1}+\gamma_{4} \delta_{m+1}^{n+1}+\gamma_{5} \delta_{m+2}^{n+1} \\
& =\gamma_{5} \delta_{m-2}^{n+1}+\gamma_{4} \delta_{m-1}^{n}+\gamma_{3} \delta_{m}^{n}+\gamma_{2} \delta_{m+1}^{n}+\gamma_{1} \delta_{m+2}^{n}
\end{aligned}
$$

where

$$
\begin{aligned}
& \gamma_{1}=(1-E-M), \quad \gamma_{2}=(26-10 E-2 M), \\
& \gamma_{3}=(66+6 M), \quad \gamma_{4}=(26+10 E-2 M), \\
& \gamma_{5}=(1+E-M), \\
& m=0,1, \ldots, N, \quad E=\left(1+6 Z_{m}\right) \frac{5}{2 h} \Delta t, \quad M=\frac{20}{h^{2}} \mu .
\end{aligned}
$$

Substituting the Fourier mode (15) into (19) gives the growth factor $g$ of the form

$$
g=\frac{a-i b}{a+i b}
$$

where

$$
\begin{aligned}
& a=33+3 M+(26-2 M) \cos [h k]+(1-M) \cos [2 h k], \\
& b=10 E \sin [h k]+E \sin [2 h k] .
\end{aligned}
$$

The modulus of $|g|$ is 1 , therefore the linearized scheme is unconditionally stable.

\section{Results and discussion}

In this section, we consider the following four test problems: the motion of a single solitary wave, the interaction of two and three solitary waves and the Maxwellian initial condition. Accuracy and efficiency of the method are measured by the error norms $L_{2}$

$$
L_{2}=\left\|U^{\text {exact }}-U_{N}\right\|_{2} \simeq \sqrt{h \sum_{J=1}^{N}\left|U_{j}^{\text {exact }}-\left(U_{N}\right)_{j}\right|^{2}},
$$


and $L_{\infty}$

$$
L_{\infty}=\left\|U^{\text {exact }}-U_{N}\right\|_{\infty} \simeq \max _{j}\left|U_{j}^{\text {exact }}-\left(U_{N}\right)_{j}\right|, \quad j=1,2, \ldots, N-1
$$

The MRLW equation satisfies only three conservation laws given by [38]

$$
\begin{aligned}
& I_{1}=\int_{a}^{b} U d x \simeq h \sum_{j=1}^{N} U_{j}^{n}, \\
& I_{2}=\int_{a}^{b}\left[U^{2}+\mu\left(U_{x}\right)^{2}\right] d x \simeq h \sum_{j=1}^{N}\left[\left(U_{j}^{n}\right)^{2}+\mu\left(U_{x}\right)_{j}^{n}\right], \\
& I_{3}=\int_{a}^{b}\left(U^{4}-\mu U_{x}^{2}\right) d x \simeq h \sum_{j=1}^{N}\left[\left(U_{j}^{n}\right)^{4}-\mu\left(U_{x}\right)_{j}^{n}\right],
\end{aligned}
$$

which correspond to conversation of mass, momentum and energy, respectively. In the simulation of a solitary wave motion, the invariants $I_{1}, I_{2}$ and $I_{3}$ are monitored to check the conversation of the numerical algorithm.

\subsection{The motion of a single solitary wave}

For this problem, MRLW Eq. (2) is considered with the boundary condition $U \rightarrow 0$ as $x \rightarrow \pm \infty$ and the initial condition

$$
U(x, 0)=\sqrt{c} \operatorname{sech}\left(p\left(x-x_{0}\right)\right)
$$

Note that the analytical solution of this problem can be written as

$$
U(x, t)=\sqrt{c} \operatorname{sech}\left(p\left(x-(c+1) t-x_{0}\right)\right)
$$

where $p=\sqrt{\frac{c}{\mu(c+1)}}, x_{0}$ and $c$ are arbitrary constants. The constants of motion, for a solitary wave of amplitude $\sqrt{c}$ and width depending on $p$ may be evaluated analytically as [29]

$$
\begin{aligned}
& I_{1}=\int_{-\infty}^{\infty} U(x, 0) d x=\frac{\pi \sqrt{c}}{p}, \\
& I_{2}=\int_{-\infty}^{\infty}\left(U^{2}(x, 0)+\mu U_{x}^{2}(x, 0)\right) d x=\frac{2 c}{p}+\frac{2 \mu p c}{3}, \\
& I_{3}=\int_{-\infty}^{\infty}\left(U^{4}(x, 0)-\mu U_{x}^{2}(x, 0)\right) d x=\frac{4 c^{2}}{3 p}-\frac{2 \mu p c}{3} .
\end{aligned}
$$

For our computational work, we have chosen two sets of parameters. Firstly, we have used the parameters $c=1, \mu=1, h=0.2, x_{0}=40, k=0.025$ over the interval $[0,100]$ to coincide with those of earlier papers [28-30,35]. So, the solitary wave has amplitude 1.0 and the computations are done up to time $t=10$ to obtain the invariants and error norms $L_{2}$ and $L_{\infty}$ at various times. Error norms $L_{2}, L_{\infty}$ and three invariants of the MRLW equation are listed in Table 1. It is seen that the error norms are found to be small enough 
Table 1 Invariants and error norms for a single solitary wave with $c=1, h=0.2, k=0.025$, $0 \leq x \leq 100$

\begin{tabular}{rlllll}
\hline $\boldsymbol{t}$ & $\boldsymbol{I}_{\mathbf{1}}$ & $\boldsymbol{I}_{\mathbf{2}}$ & $\boldsymbol{I}_{\mathbf{3}}$ & $\boldsymbol{L}_{\mathbf{2}} \times \mathbf{1 0}^{\mathbf{3}}$ & $\boldsymbol{L}_{\boldsymbol{\infty}} \mathbf{\times} \mathbf{1 0}^{\mathbf{3}}$ \\
\hline 0 & 4.4428660 & 3.2998226 & 1.4142046 & 0.00000000 & 0.000000000 \\
1 & 4.4428660 & 3.2998068 & 1.4142204 & 0.28867055 & 0.17189210 \\
2 & 4.4428660 & 3.2997776 & 1.4142496 & 0.56818930 & 0.32423631 \\
3 & 4.4428660 & 3.2997536 & 1.4142736 & 0.83577886 & 0.46169463 \\
4 & 4.4428660 & 3.2997375 & 1.4142897 & 1.09458149 & 0.59234819 \\
5 & 4.4428660 & 3.2997272 & 1.4143000 & 1.34807894 & 0.72040575 \\
6 & 4.4428660 & 3.2997206 & 1.4143066 & 1.59852566 & 0.84732049 \\
7 & 4.4428660 & 3.2997164 & 1.4143108 & 1.84722430 & 0.97368288 \\
8 & 4.4428660 & 3.2997137 & 1.4143135 & 2.09491698 & 1.09976163 \\
9 & 4.4428661 & 3.2997119 & 1.4143153 & 2.34203425 & 1.22568849 \\
10 & 4.4428661 & 3.2997108 & 1.4143165 & 2.58891199 & 1.35164457 \\
\hline
\end{tabular}

Table 2 Errors and invariants for a single solitary wave with $c=1, h=0.2, k=0.025$, $0 \leq x \leq 100$, at $t=10$

\begin{tabular}{llllcc}
\hline Method & $\boldsymbol{I}_{\mathbf{1}}$ & $\boldsymbol{I}_{\mathbf{2}}$ & $\boldsymbol{I}_{\mathbf{3}}$ & $\mathbf{L}_{\mathbf{2}} \times \mathbf{1 0}^{\mathbf{3}}$ & $\boldsymbol{L}_{\boldsymbol{\infty}} \times \mathbf{1 0}^{\mathbf{3}}$ \\
\hline Analytical & 4.4428829 & 3.2998316 & 1.4142135 & 0 & 0 \\
Present & 4.4428661 & 3.2997108 & 1.4143165 & 2.58891 & 1.35164 \\
Pet-Gal.[28] & 4.44288 & 3.29981 & 1.41416 & 3.00533 & 1.68749 \\
Cubic B-splines coll-CN[29] & 4.442 & 3.299 & 1.413 & 16.39 & 9.24 \\
Cubic B-splines coll+PA-CN[29] & 4.440 & 3.296 & 1.411 & 20.3 & 11.2 \\
Cubic B-splines coll[30] & 4.44288 & 3.29983 & 1.41420 & 9.30196 & 5.43718 \\
MQ[35] & 4.4428829 & 3.29978 & 1.414163 & 3.914 & 2.019 \\
IMQ[35] & 4.4428611 & 3.29978 & 1.414163 & 3.914 & 2.019 \\
IQ[35] & 4.4428794 & 3.29978 & 1.414163 & 3.914 & 2.019 \\
GA[35] & 4.4428829 & 3.29978 & 1.414163 & 3.914 & 2.019 \\
TPS[35] & 4.4428821 & 3.29972 & 1.414104 & 4.428 & 2.306 \\
\hline
\end{tabular}

and the computed values of invariants are in good agreement with their analytical values $I_{1}=4.4428829, I_{2}=3.2998316, I_{3}=1.4142135$. Percentage values of the relative error of the conserved quantities $I_{1}, I_{2}$ and $I_{3}$ are calculated with respect to the conserved quantities at $t=0$. Percentage values of relative changes of $I_{1}, I_{2}$ and $I_{3}$ are found to be $0.001 \times 10^{-3} \%, 3.389 \times 10^{-3} \%, 7.909 \times 10^{-3} \%$, respectively. Thus, the invariants remain almost constant during the computer run. Table 2 displays a comparison of the values of the invariants and error norms obtained by the present method with those obtained by other methods [28-30,35]. It can be seen from Table 2 that the error norms obtained by the present method are smaller than other methods [28-30,35]. Figure 1 shows the motion of a solitary wave with $c=1, h=0.2, k=0.025$ at different time levels. It is observed that the soliton moves to the right at a constant speed and almost unchanged amplitude with increasing time, as expected. At $t=0$ the amplitude is 1.0 which is located at $x=40$, while it is 0.999946 which is located at $x=60$. At times $t=0$ and $t=10$, the absolute difference in amplitude is $5 \times 10^{-5}$ so there is a little change between the amplitudes.

For the second set, the parameters $\mu=1, c=0.3, h=0.1, k=0.01$ and $x_{0}=40$ with range $[0,100]$ are chosen to compare the results obtained by the present method with those obtained given in Refs. [28, 30, 32, 34, 35]. So, the solitary wave has amplitude 0.547723 and the computations are done up to time $t=20$ to obtain the invariants and error norms $L_{2}$ and $L_{\infty}$ at various times. Error norms $L_{2}$ and $L_{\infty}$ and conserved quantities are tabulated in Table 3 together with the results obtained with Refs. [28, 30, 32, 34, 35]. As it is seen from the table, the error norms obtained by the present method are smaller than those 


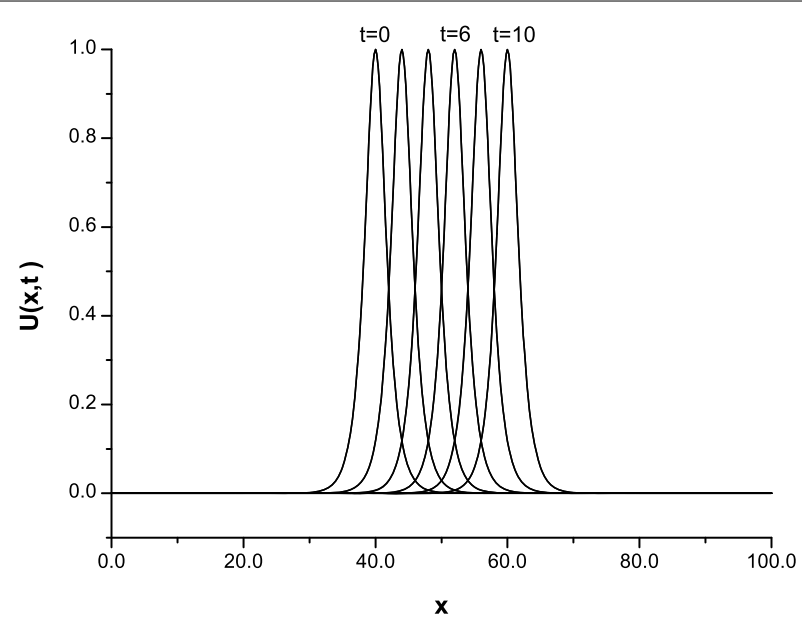

Figure 1 Single solitary wave with $c=1, h=0.2, \Delta t=0.025,0 \leq x \leq 100, t=0,2,4,6,8$ and 10 .

given in Refs. [30, 32] and almost the same as those in Refs. [28, 34, 35]. The agreement between numerical and analytic solutions is perfect which is given by Eq. 23. Percentage values of relative changes of $I_{1}, I_{2}$ and $I_{3}$ are found to be $0.001 \times 10^{-3} \%, 0.246 \times 10^{-3} \%$, $2.152 \times 10^{-3} \%$, respectively. Moreover, from Table 3 , the changing of the invariants $I_{1}, I_{2}$ and $I_{3}$ during the computer run is less than $1 \times 10^{-7}, 3.3 \times 10^{-6}, 3.3 \times 10^{-6}$, respectively. The profiles of the solitary wave at different time levels have been shown in Figure 2. The distributions of the errors at time $t=10$ and $t=20$ are shown graphically for solitary wave amplitudes 1 and 0.3 in Figure 3. It is seen that the maximum errors are about at the tip of the solitary waves and between $-6 \times 10^{-3}$ and $6 \times 10^{-3},-2 \times 10^{-4}$ and $2 \times 10^{-4}$, respectively.

\subsection{Interaction of two solitary waves}

Here the interaction of two solitary waves is studied by using the initial condition given by the linear sum of two well-separated solitary waves having various amplitudes

$$
U(x, 0)=\sum_{j=1}^{2} A_{j} \operatorname{sech}\left(p_{j}\left(x-x_{j}\right)\right)
$$

where $A_{j}=\sqrt{c_{j}}, p_{j}=\sqrt{\frac{c_{j}}{\mu\left(c_{j}+1\right)}}, j=1,2, c_{j}$ and $x_{j}$ are arbitrary constants. The analytical values of the invariants are found by [29]

$$
\begin{aligned}
& I_{1}=\sum_{j=1}^{2} \frac{\pi \sqrt{c_{j}}}{p_{j}} \\
& I_{2}=\sum_{j=1}^{2}\left(\frac{2 c_{j}}{p_{j}}+\frac{2 \mu p_{j} c_{j}}{3}\right), \\
& I_{3}=\sum_{j=1}^{2}\left(\frac{4 c_{j}^{2}}{3 p_{j}}-\frac{2 \mu p_{j} c_{j}}{3}\right) .
\end{aligned}
$$


Table 3 Invariants and error norms for a single solitary wave with $c=0.3, h=0.1, k=0.01$, $0 \leq x \leq 100$

\begin{tabular}{|c|c|c|c|c|c|}
\hline$t$ & $I_{1}$ & $I_{2}$ & $I_{3}$ & $L_{2} \times 10^{4}$ & $L_{\infty} \times 10^{4}$ \\
\hline 0 & 3.5820205 & 1.3450941 & 0.1537283 & 0.0000000 & 0.0000000 \\
\hline 2 & 3.5820205 & 1.3450944 & 0.1537280 & 0.0082694 & 0.0034843 \\
\hline 4 & 3.5820205 & 1.3450950 & 0.1537274 & 0.0162937 & 0.0070162 \\
\hline 6 & 3.5820206 & 1.3450955 & 0.1537268 & 0.0242346 & 0.0105732 \\
\hline 8 & 3.5820206 & 1.3450960 & 0.1537264 & 0.0322064 & 0.0141521 \\
\hline 10 & 3.5820206 & 1.3450964 & 0.1537260 & 0.0402374 & 0.0177376 \\
\hline 12 & 3.5820206 & 1.3450966 & 0.1537257 & 0.0483276 & 0.0213278 \\
\hline 14 & 3.5820206 & 1.3450969 & 0.1537255 & 0.0564695 & 0.0249138 \\
\hline 16 & 3.5820206 & 1.3450971 & 0.1537253 & 0.0646548 & 0.0285146 \\
\hline 18 & 3.5820206 & 1.3450972 & 0.1537251 & 0.0728758 & 0.0321067 \\
\hline 20 & 3.5820204 & 1.3450974 & 0.1537250 & 0.8112594 & 0.3569076 \\
\hline $20[30]$ & 3.58197 & 1.34508 & 0.153723 & 6.06885 & 2.96650 \\
\hline $20[34]$ & 3.581967 & 1.345076 & 0.153723 & 0.508927 & 0.222284 \\
\hline 20[35]MQ & 3.5819665 & 1.3450764 & 0.153723 & 0.51498 & 0.22551 \\
\hline 20[35]IMQ & 3.5819664 & 1.3450764 & 0.153723 & 0.51498 & 0.22551 \\
\hline 20[35]।Q & 3.5819654 & 1.3450764 & 0.153723 & 0.51498 & 0.22551 \\
\hline $20[35] \mathrm{GA}$ & 3.5819665 & 1.3450764 & 0.153723 & 0.51498 & 0.22551 \\
\hline 20[35]TPS & 3.5819663 & 1.3450759 & 0.153723 & 0.51498 & 0.26605 \\
\hline
\end{tabular}

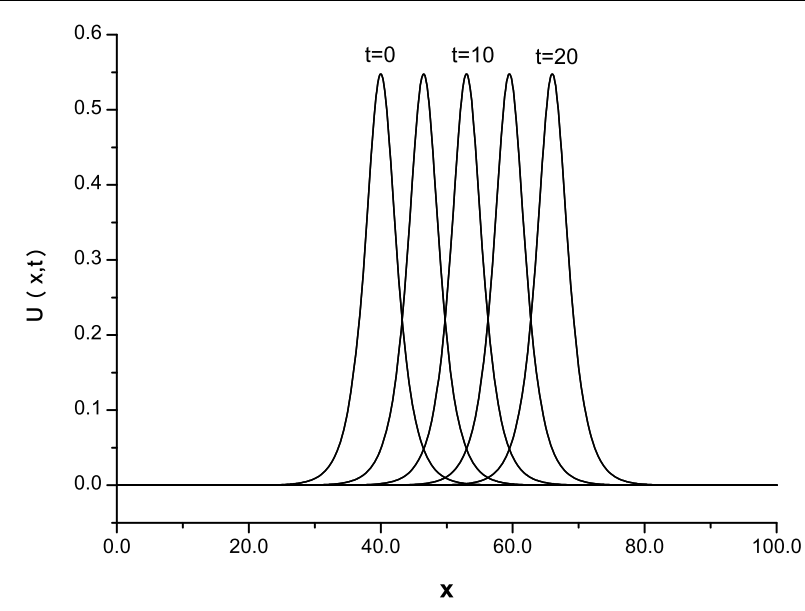

Figure 2 Single solitary wave with $c=0.3, h=0.1, \Delta t=0.01,0 \leq x \leq 100$ at times $t=0,5,10,15$ and 20.

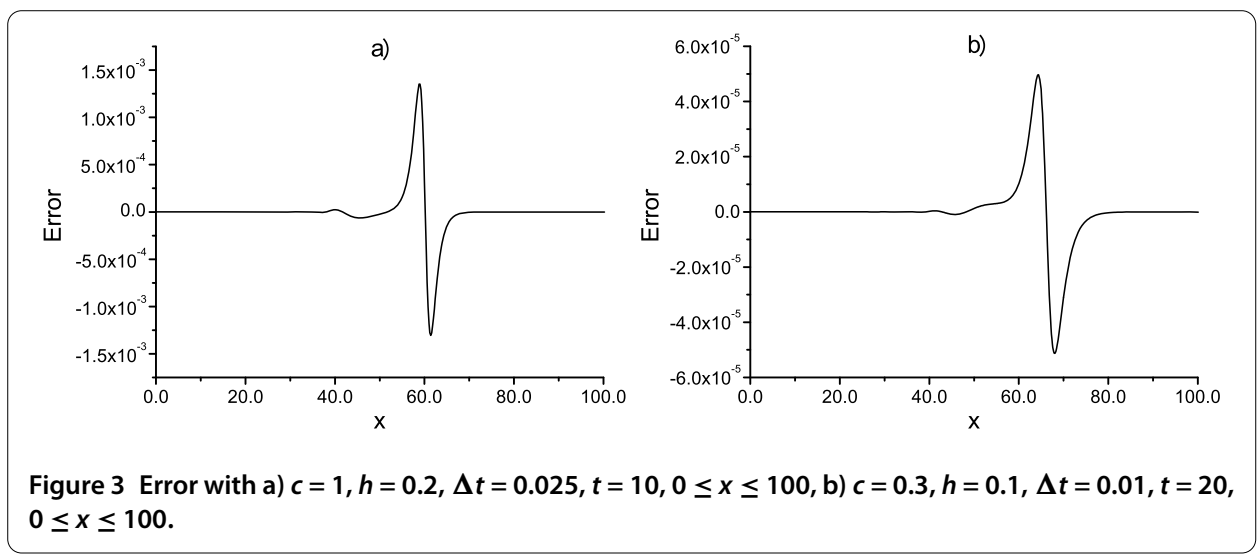


Table 4 Comparison of invariants for the interaction of two solitary waves with results from [34] with $h=0.2, k=0.025$ in the region $0 \leq x \leq 250$

\begin{tabular}{|c|c|c|c|c|c|c|}
\hline \multicolumn{4}{|c|}{ Present method } & \multicolumn{3}{|l|}{ [34] } \\
\hline$t$ & $I_{1}$ & $I_{2}$ & $I_{3}$ & $I_{1}$ & $I_{2}$ & $I_{3}$ \\
\hline 0 & 11.4676542 & 14.6292080 & 22.8803584 & 11.467698 & 14.629277 & 22.880432 \\
\hline 2 & 11.4678169 & 14.6282301 & 22.8813363 & 11.467698 & 14.624259 & 22.860365 \\
\hline 4 & 11.4679819 & 14.6282293 & 22.8813371 & 11.467698 & 14.619226 & 22.840279 \\
\hline 6 & 11.4681349 & 14.6181053 & 22.8914611 & 11.467699 & 14.614169 & 22.820069 \\
\hline 8 & 11.4675390 & 14.1393389 & 23.3702275 & 11.467700 & 14.606821 & 22.787857 \\
\hline 10 & 11.4674118 & 14.0502062 & 23.4593602 & 11.467700 & 14.603687 & 22.771773 \\
\hline 12 & 11.4685494 & 14.6816556 & 22.8279107 & 11.467699 & 14.603056 & 22.775766 \\
\hline 14 & 11.4687073 & 14.6648742 & 22.8446922 & 11.467699 & 14.598059 & 22.756029 \\
\hline 16 & 11.4688627 & 14.6459207 & 22.8636457 & 11.467700 & 14.593048 & 22.736127 \\
\hline 18 & 11.4690242 & 14.6370095 & 22.8725569 & 11.467700 & 14.588061 & 22.716289 \\
\hline 20 & 11.4691886 & 14.6331334 & 22.8764330 & 11.467701 & 14.583089 & 22.696510 \\
\hline $20[28]$ & 11.4677 & 14.6299 & 22.8806 & & & \\
\hline $20[30]$ & 11.4677 & 14.6292 & 22.8809 & & & \\
\hline 20[35]MQ & 11.467698 & 14.583052 & 22.696539 & & & \\
\hline 20[35]IMQ & 11.467679 & 14.583052 & 22.696539 & & & \\
\hline 20[35]IQ & 11.467690 & 14.583052 & 22.696539 & & & \\
\hline $20[35] \mathrm{GA}$ & 11.467698 & 14.583052 & 22.696539 & & & \\
\hline 20[35]TPS & 11.467742 & 14.582424 & 22.694269 & & & \\
\hline
\end{tabular}

For the numerical simulation, the parameters $\mu=1, h=0.2, k=0.025, c_{1}=4, c_{2}=1$, $x_{1}=25, x_{2}=55$ are used over the range $0 \leq x \leq 250$ to coincide with those used by Refs. $[28,30,34,35]$. The experiment is run from $t=0$ to $t=20$ and the values of invariant quantities $I_{1}, I_{2}$ and $I_{3}$ are recorded in Table 4 . The analytical values of the invariants for this case are $I_{1}=11.467698, I_{2}=14.629243, I_{3}=22.880466$. A comparison of the values of the invariants obtained by the present method with those obtained in Refs. [28, 30, 34,35 ] are listed in Table 4. It is seen that the obtained values of the invariants remain almost constant during the computer run. The development of the interaction of two solitary waves is shown in Figure 4. It can be seen from the figure that at $t=0$ the wave with larger amplitude is to the left of the second wave with smaller amplitude. Since the taller wave moves faster than the shorter one, it catches up and collides with the shorter one at $t=8$ and then moves away from the shorter one as time increases. At $t=20$, the amplitude of larger waves is 2.001090 at the point $x=127.4$ whereas the amplitude of the smaller one is 0.996399 at the point $x=92$. It is found that the absolute difference in amplitude is $3.60 \times 10^{-3}$ for the smaller wave and $1.09 \times 10^{-3}$ for the larger wave for this algorithm.

\subsection{Interaction of three solitary waves}

For this problem, the behavior of interaction of three solitary waves having different amplitudes and traveling in the same direction is studied. So, we consider Eq. (2) with the initial condition given by the linear sum of three well-separated solitary waves of different amplitudes

$$
U(x, 0)=\sum_{j=1}^{3} A_{j} \operatorname{sech}\left(p_{j}\left(x-x_{j}\right)\right)
$$



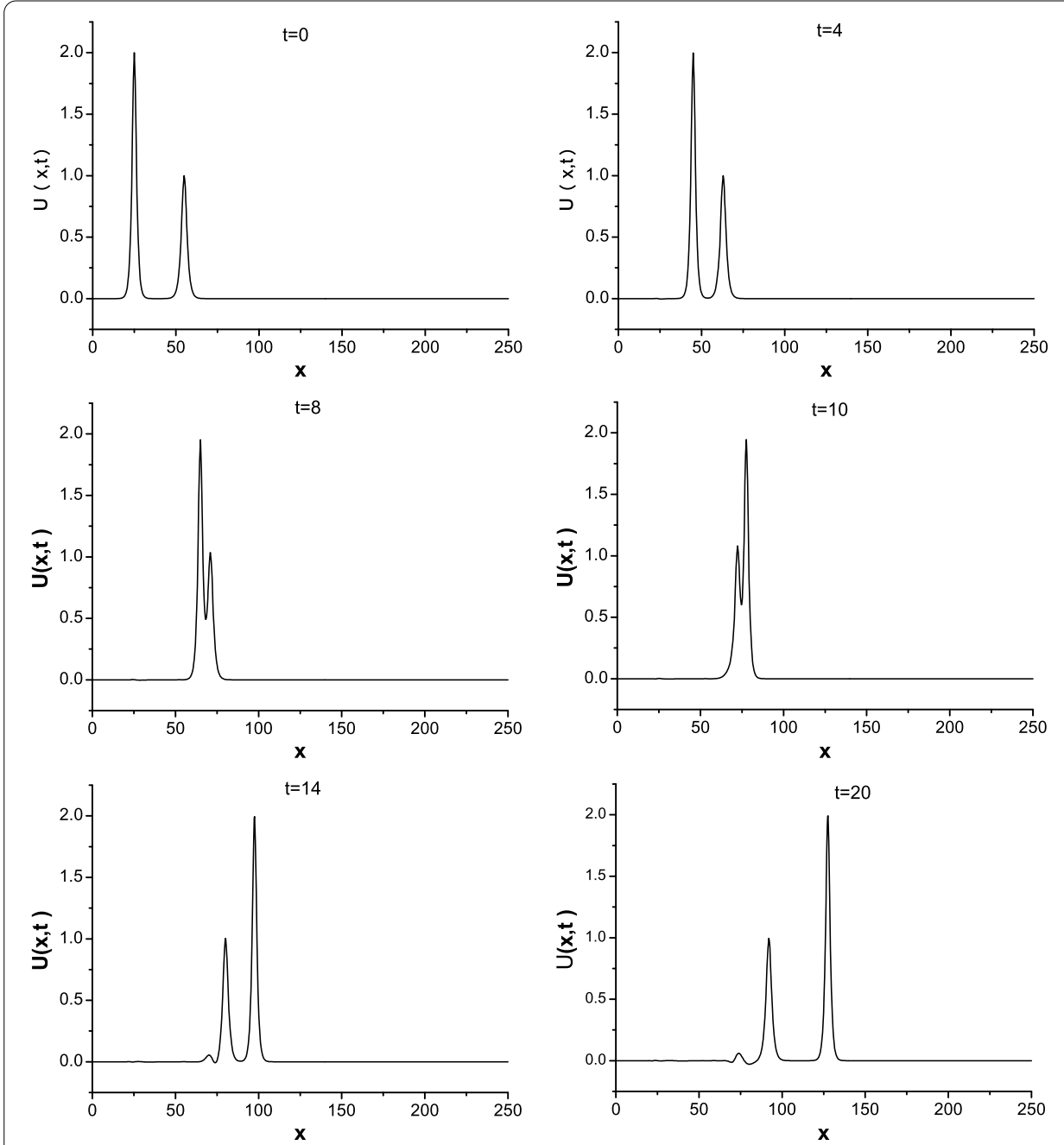

Figure 4 Interaction of two solitary waves with $t=0,4,8,10,14,20$.

where $A_{j}=\sqrt{c_{j}}, p_{j}=\sqrt{\frac{c_{j}}{\mu\left(c_{j}+1\right)}}, j=1,2,3, c_{j}$ and $x_{j}$ are arbitrary constants. The analytical values of the conservation laws are found from Eq. (23) as follows:

$$
\begin{aligned}
& I_{1}=\sum_{j=1}^{3} \frac{\pi \sqrt{c_{j}}}{p_{j}}, \\
& I_{2}=\sum_{j=1}^{3}\left(\frac{2 c_{j}}{p_{j}}+\frac{2 \mu p_{j} c_{j}}{3}\right), \\
& I_{3}=\sum_{j=1}^{3}\left(\frac{4 c_{j}^{2}}{3 p_{j}}-\frac{2 \mu p_{j} c_{j}}{3}\right) .
\end{aligned}
$$

For the purpose of comparison, parameters $\mu=1, h=0.2, k=0.025, c_{1}=4, c_{2}=1$, $c_{3}=0.25, x_{1}=15, x_{2}=45, x_{3}=60$ are used over the region $0 \leq x \leq 250$. During the simulation, time is taken up to $t=45$. The analytical values of the invariants for this case are $I_{1}=14.9801, I_{2}=15.8218, I_{3}=22.9923$. A comparison of the values of the invariants 
Table 5 Comparison of invariants for the interaction of three solitary waves with results from [34] with $h=0.2, k=0.025$ in the region $0 \leq x \leq 250$

\begin{tabular}{|c|c|c|c|c|c|c|}
\hline \multicolumn{4}{|c|}{ Present method } & \multicolumn{3}{|l|}{ [34] } \\
\hline$t$ & $I_{1}$ & $I_{2}$ & $I_{3}$ & $I_{1}$ & $I_{2}$ & $/ 3$ \\
\hline 0 & 14.9800762 & 15.8374849 & 23.0081806 & 14.980099 & 15.837528 & 23.008136 \\
\hline 5 & 14.9381371 & 15.7382326 & 23.1074329 & 14.980105 & 15.824928 & 22.957891 \\
\hline 10 & 14.9071292 & 14.1781087 & 24.6675567 & 14.980109 & 15.807025 & 22.877972 \\
\hline 15 & 14.8836886 & 15.3648852 & 23.4807802 & 14.980106 & 15.807032 & 22.885947 \\
\hline 20 & 14.8503851 & 15.5659364 & 23.2797291 & 14.980106 & 15.795022 & 22.837454 \\
\hline 25 & 14.8194163 & 15.6235556 & 23.2221098 & 14.980107 & 15.782840 & 22.788852 \\
\hline 30 & 14.7905616 & 15.5976717 & 23.2479938 & 14.980107 & 15.770634 & 22.740419 \\
\hline 35 & 14.7636015 & 15.5610664 & 23.2845991 & 14.980108 & 15.758480 & 22.692279 \\
\hline 40 & 14.7383184 & 15.5256320 & 23.3200335 & 14.980108 & 15.746389 & 22.644448 \\
\hline 45 & 14.7145273 & 15.4927592 & 23.3529062 & 14.968030 & 15.734374 & 22.596591 \\
\hline $45[30]$ & 13.7043 & 15.6563 & 22.9303 & & & \\
\hline 45[35]MQ & 14.96814 & 15.73434 & 22.596625 & & & \\
\hline 45[35] IMQ & 14.96808 & 15.73434 & 22.596625 & & & \\
\hline 45[35]IQ & 14.96813 & 15.73434 & 22.596625 & & & \\
\hline $45[35] \mathrm{GA}$ & 14.96810 & 15.73433 & 22.596626 & & & \\
\hline 45[35]TPS & 14.96824 & 15.73376 & 22.594494 & & & \\
\hline
\end{tabular}

obtained by the present method with those obtained in Refs. [30, 34, 35] are shown in Table 5 . It is observed from the table that the obtained values of the invariants remain almost constant during the computer run which are all in good agreement with their analytical values given by Eq. (27). The absolute difference between the values of the conservative constants obtained by the present method at times $t=0$ and $t=45$ are $\Delta I_{1}=2.6 \times 10^{-1}$, $\Delta I_{2}=3.4 \times 10^{-1}, \Delta I_{3}=3.4 \times 10^{-3}$, respectively. Figure 5 shows the interaction of these solitary waves at different times. As it is seen from the Figure 5 , the interaction started at about time $t=10$, overlapping processes occurred between time $t=15$ and $t=40$ and waves started to resume their original shapes after the time $t=40$.

\subsection{The Maxwellian initial condition}

Finally, we have studied the development of the Maxwellian initial condition

$$
U(x, 0)=\exp \left(-(x-40)^{2}\right)
$$

into a train of solitary waves. As it is known, with the Maxwellian condition (28), the behavior of the solution depends on the values of $\mu$. We study each of the following cases: $\mu=0.1, \mu=0.04, \mu=0.015$ and $\mu=0.01$. For $\mu=0.1$, only a single soliton is formed as shown in Figure 6a. When $\mu=0.04$ and $\mu=0.015$, two and three stable solitons are formed, respectively, as shown in Figure 6b, c. For $\mu=0.01$, the Maxwellian initial condition has decayed into four solitary waves as shown in Figure 6d. All figures were drawn up at time $t=14.5$. The peaks of the well-developed wave lie on a straight line, so that their velocities are linearly dependent on their amplitudes. We also observe a small oscillating tail appearing behind the last wave in all Maxwellian figures. The obtained numerical values of the invariants are given in Table 6.

\section{Conclusions}

A numerical solution of the MRLW equation based on the quintic B-spline finite element has been successfully presented. The nonlinear term of the equation is linearized by using 

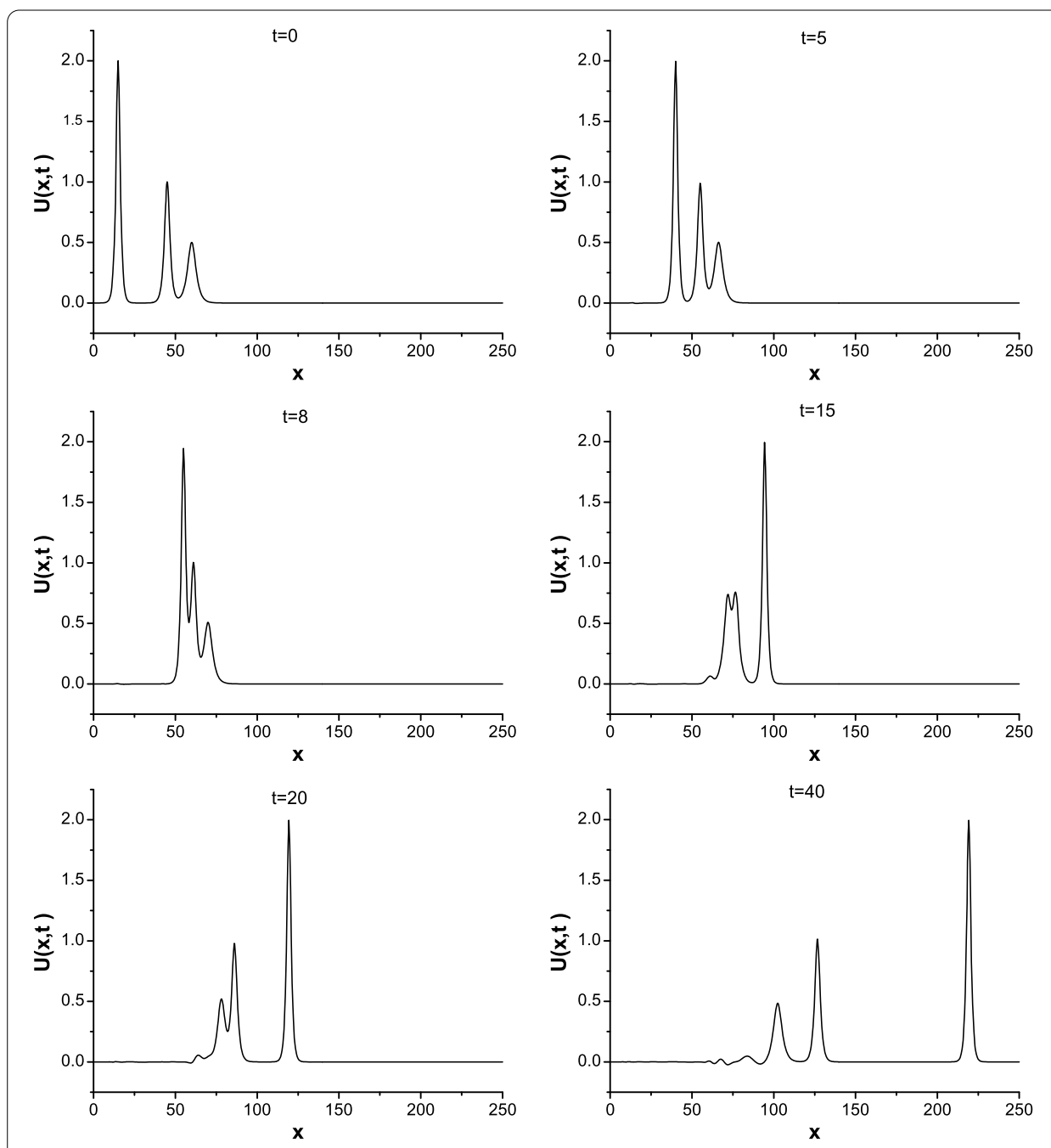

Figure 5 Interaction of three solitary waves with $t=0,5,8,15,20,40$.

Table 6 Invariants of the MRLW equation using the Maxwellian initial condition

\begin{tabular}{|c|c|c|c|c|c|c|c|c|}
\hline$t$ & $\mu$ & $I_{1}$ & $I_{2}$ & $I_{3}$ & $\mu$ & $I_{1}$ & $I_{2}$ & $I_{3}$ \\
\hline 0 & 0.1 & 1.7724809 & 1.3786633 & 0.7609104 & 0.015 & 1.7724809 & 1.2721327 & 0.8674410 \\
\hline 3 & & 1.7721927 & 1.4728222 & 0.6667515 & & 1.7436793 & 1.4180550 & 0.7215188 \\
\hline 6 & & 1.7717480 & 1.4720618 & 0.6675119 & & 1.7244620 & 1.4036726 & 0.7359011 \\
\hline 9 & & 1.7713064 & 1.4715473 & 0.6680264 & & 1.7116986 & 1.3940073 & 0.7455664 \\
\hline 12 & & 1.7708674 & 1.4711193 & 0.6684544 & & 1.7021509 & 1.3870669 & 0.7525068 \\
\hline 15 & & 1.7704309 & 1.4707290 & 0.6688447 & & 1.6945087 & 1.3816736 & 0.7579001 \\
\hline 0 & 0.01 & 1.7724809 & 1.2658662 & 0.8737075 & 0.04 & 1.7724809 & 1.3034651 & 0.8361087 \\
\hline 3 & & 1.7264258 & 1.4001430 & 0.7394307 & & 1.7685556 & 1.4501960 & 0.6893777 \\
\hline 6 & & 1.7031386 & 1.3850514 & 0.7545223 & & 1.7637294 & 1.4456393 & 0.6939344 \\
\hline 9 & & 1.6885258 & 1.3755380 & 0.7640357 & & 1.7592882 & 1.4415131 & 0.6980606 \\
\hline 12 & & 1.6777156 & 1.3686563 & 0.7709174 & & 1.7551780 & 1.4378815 & 0.7016922 \\
\hline 15 & & 1.6691476 & 1.3635304 & 0.7760434 & & 1.7513552 & 1.4345989 & 0.7049748 \\
\hline
\end{tabular}

a form given in the paper [36]. Four test problems are studied to examine the performance of the scheme. To show how good and accurate the numerical solutions of the test problems are, the error norms $L_{2}$ and $L_{\infty}$ and the invariant quantities $I_{1}, I_{2}$ and $I_{3}$ have 

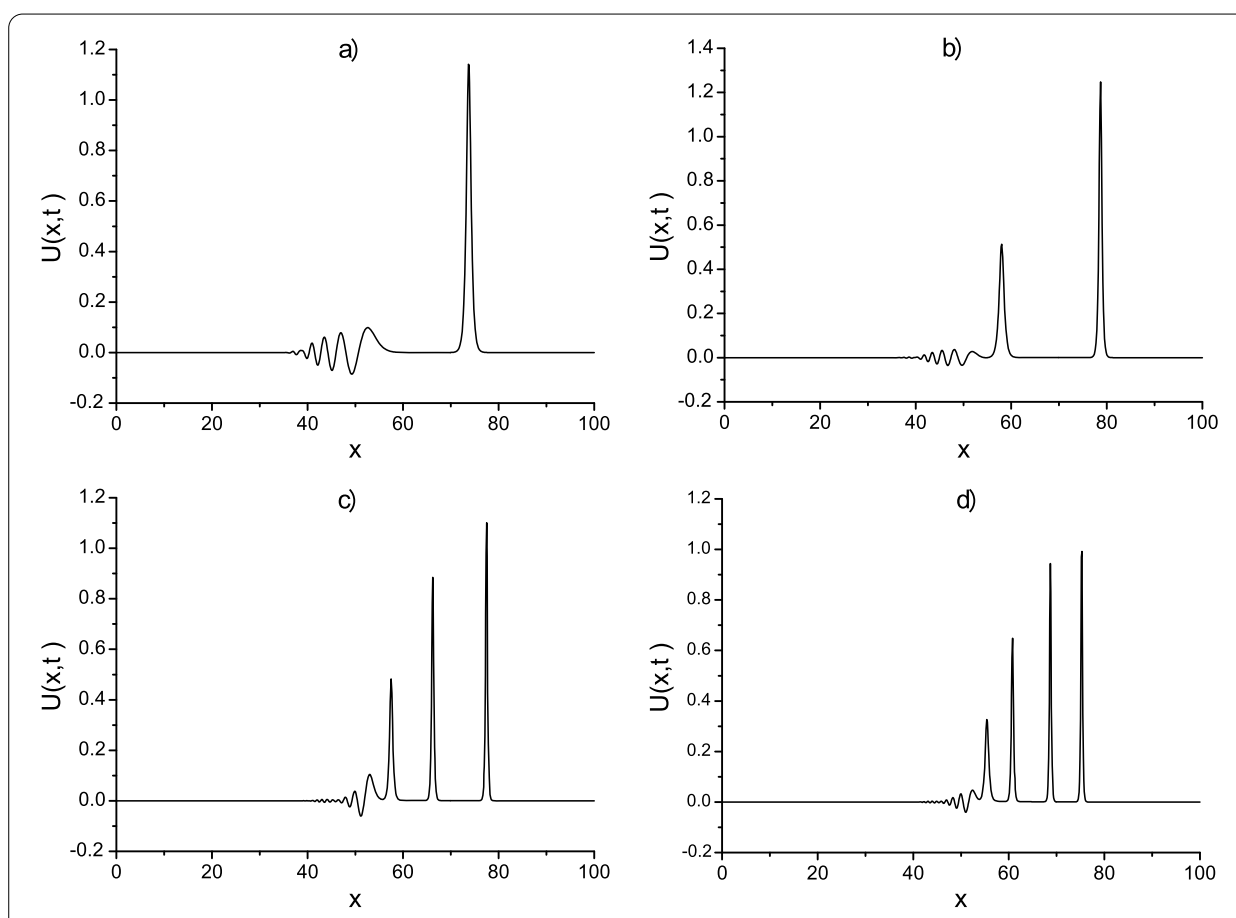

Figure 6 Maxwellian initial condition at $t=14.5$ with a) $\mu=0.1$, b) $\mu=0.04$, c) $\mu=0.015$, d) $\mu=0.01$.

been used. It is seen that the error norms are sufficiently small and the invariants are well conserved. The method successfully models the motion and interaction of solitary waves. The computed results indicate that the present method is more accurate than some earlier results found in the literature. So, it can be said that the method is a reliable one for obtaining the numerical solutions of a wider range of physically important non-linear partial differential equations.

Competing interests

The authors declare that they have no competing interests.

Authors' contributions

All the three authors have almost equal contributions to the article. In particular, SBGK participated in the design of the basic outline of the article and equations. NMY participated in the application of the method and obtaining the iterative formulae. YU participated in coding and running the necessary programs. All authors worked together to check and test the programs, to obtain the results, to carry out the literature search. All authors read, checked, corrected and approved the final manuscript.

\section{Author details}

${ }^{1}$ Department of Mathematics, Faculty of Science and Art, Nevsehir University, Nevsehir, 50300, Turkey. ${ }^{2}$ Department of Mathematics, Faculty of Science and Art, Inönü University, Malatya, 44280, Turkey.

\section{Acknowledgements}

Dedicated to Professor Hari M Srivastava.

The authors would like to thank the reviewers for their careful reading and making some useful comments which improved the presentation of the paper.

Received: 19 November 2012 Accepted: 29 January 2013 Published: 14 February 2013

\section{References}

1. Peregrine, DH: Calculations of the development of an undular bore. J. Fluid Mech. 25, 321-330 (1966)

2. Bona, JL, Pryant, PJ: A mathematical model for long wave generated by wave makers in nonlinear dispersive systems. Proc. Camb. Philos. Soc. 73, 391-405 (1973)

3. Benjamin, TB, Bona, JL, Mahoney, JL: Model equations for long waves in nonlinear dispersive media. Philos. Trans. R. Soc. Lond. 272, 47-78 (1972) 
4. Morrison, PJ, Meiss, JD, Cary, JA: Scattering of regularized long wave solitary waves. Physica 11D, 324-336 (1984)

5. Eilbeck, JC, McGuire, GR: Numerical study of the regularized long wave equation, II: Interaction of solitary wave. J. Comput. Phys. 23, 63-73 (1977)

6. Jain, PC, Shankar, R, Singh, TV: Numerical solution of regularized long wave equation. Commun. Numer. Methods Eng. 9, 579-586 (1993)

7. Bhardwaj, D, Shankar, R: A computational method for regularized long wave equation. Comput. Math. Appl. 40 1397-1404 (2000)

8. Chang, Q, Wang, G, Guo, B: Conservative scheme for a model of nonlinear dispersive waves and its solitary waves induced by boundary motion. J. Comput. Phys. 93, 360-375 (1995)

9. Gardner, LRT, Gardner, GA: Solitary waves of the regularized long wave equation. J. Comput. Phys. 91, 441-459 (1990)

10. Gardner, LRT, Gardner, GA, Dogan, A: A least-squares finite element scheme for the RLW equation. Commun. Numer. Methods Eng. 12, 795-804 (1996)

11. Gardner, LRT, Gardner, GA, Dag, I: A B-spline finite element method for the regularized long wave equation. Commun. Numer. Methods Eng. 11, 59-68 (1995)

12. Alexander, ME, Morris, JL: Galerkin method applied to some model equations for nonlinear dispersive waves. J. Comput. Phys. 30, 428-451 (1979)

13. Serna, JMS, Christie, I: Petrov Galerkin methods for nonlinear dispersive wave. J. Comput. Phys. 39, 94-102 (1981)

14. Dogan, A: Numerical solution of RLW equation using linear finite elements within Galerkin's method. Appl. Math. Model. 26, 771-783 (2002)

15. Esen, A, Kutluay, S: Application of lumped Galerkin method to the regularized long wave equation. Appl. Math. Comput. 174(2), 833-845 (2006)

16. Soliman, AA, Raslan, KR: Collocation method using quadratic b-spline for the RLW equation. Int. J. Comput. Math. 78, 399-412 (2001)

17. Soliman, AA, Hussien, MH: Collocation solution for RLW equation with septic spline. Appl. Math. Comput. 161 623-636 (2005)

18. Raslan, KR: A computational method for the regularized long wave (RLW) equation. Appl. Math. Comput. 167, 1101-1118 (2005)

19. Saka, B, Dag, I, Dogan, A: Galerkin method for the numerical solution of the RLW equation using quadratic B-splines. Int. J. Comput. Math. 81(6), 727-739 (2004)

20. Dag, I, Saka, B, Irk, D: Application of cubic B-splines for numerical solution of the RLW equation. Appl. Math. Comput 159, 373-389 (2004)

21. Dag, I, Ozer, MN: Approximation of RLW equation by least-square cubic B-spline finite element method. Appl. Math. Model. 25, 221-231 (2001)

22. Zaki, SI: Solitary waves of the splitted RLW equation. Comput. Phys. Commun. 138, 80-91 (2001)

23. Gou, BY, Cao, WM: The Fourier pseudo-spectral method with a restrain operator for the RLW equation. J. Comput. Phys. 74, 110-126 (1988)

24. Islam, S, Haq, F, Ali, A: A meshfree method for the numerical solution of the RLW equation. J. Comput. Appl. Math. 223, 997-1012 (2009)

25. Zhang, L: A finite difference scheme for generalized long wave equation. Appl. Math. Comput. 168(2), 962-972 (2005)

26. Ramos, J: Solitary wave interactions of the GRLW equation. Chaos Solitons Fractals 33, 479-491 (2007)

27. Kaya, D, El-Sayed, SM: An application of the decomposition method for the generalized KdV and RLW equations. Chaos Solitons Fractals 17, 869-877 (2003)

28. Roshan, T: A Petrov-Galerkin method for solving the generalized regularized long wave (GRLW) equation. Comput. Math. Appl. 63, 943-956 (2012)

29. Gardner, LRT, Gardner, GA, Ayoup, FA, Amein, NK: Simulations of solitary waves of the MRLW equation by B-spline finite element. Arab. J. Sci. Eng. 22, 183-193 (1997)

30. Khalifa, AK, Raslan, KR, Alzubaidi, HM: A collocation method with cubic B-splines for solving the MRLW equation. J. Comput. Appl. Math. 212, 406-418 (2008)

31. Khalifa, AK, Raslan, KR, Alzubaidi, HM: A finite difference scheme for the MRLW and solitary wave interactions. Appl. Math. Comput. 189, 346-354 (2007)

32. Raslan, KR: Numerical study of the modified regularized long wave equation. Chaos Solitons Fractals 42, 1845-1853 (2009)

33. Raslan, KR, Hassan, SM: Solitary waves for the MRLW equation. Appl. Math. Lett. 22, 984-989 (2009)

34. Haq, F, Islam, S, Tirmizi, IA: A numerical technique for solution of the MRLW equation using quartic B-splines. Appl. Math. Model. 34, 4151-4160 (2010)

35. Ali, A: Mesh free collocation method for numerical solution of initial-boundary value problems using radial basis functions. Dissertation, Ghulam Ishaq Khan Institute of Engineering Sciences and Technology (2009)

36. Rubin, SG, Graves, RA: Cubic spline approximation for problems in fluid mechanics. Nasa TR R-436, Washington DC (1975)

37. Prenter, PM: Splines and Variational Methods. Wiley, New York (1975)

38. Olver, PJ: Euler operators and conservation laws of the BBM equation. Math. Proc. Camb. Philos. Soc. 85, 143-159 (1979)

doi:10.1186/1687-2770-2013-27

Cite this article as: Karakoc et al.: Numerical approximation to a solution of the modified regularized long wave equation using quintic B-splines. Boundary Value Problems 2013 2013:27. 